\title{
El tratamiento con implantes en pacientes con diabetes. Un estudio comparativo a 7 años.
}

\section{Treatment with implants in patients with diabetes. A 7-year comparative study.}

\author{
JL Rondon Romero*, I Ortiz Garcia**, A Jimenez Guerra**, N Matos Garrido*, A España \\ Lopez*, L Monsalve Guil**, E Velasco Ortega***
}

\section{RESUMEN}

Introducción. La implantología oral puede constituir una modalidad terapéutica en el tratamiento prostodóncico de los pacientes con compromiso médico. El estudio muestra la evaluación del tratamiento con implantes en pacientes con diabetes comparados con pacientes sin diabetes.

Métodos. 48 pacientes edéntulos totales (24 pacientes diabéticos y 24 no diabéticos) fueron tratados con 96 implantes con superficie arenada y grabada Galimplant $®$ en la mandíbula para su rehabilitación prostodóncica con sobredentaduras mandibulares. 2 implantes fueron insertados en cada paciente. Los implantes fueron cargados funcionalmente tras un periodo de tiempo de 6 semanas con retenedores de fricción. Los hallazgos clínicos (implantológicos y prostodóncicos) se han seguido durante 7 años.

Resultados. Los resultados indican una supervivencia de los implantes del 95,8\%. Durante el periodo de seguimeinto clínico se perdieron 4 implantes (dos implantes en cada grupo). La pérdida de hueso marginal media fué de 0,7 $\mathrm{mm}$ en ambos grupos. El 100\% de los pacientes fueron tratados mediante una sobredentadura implantorretenida con anclajes de fricción. En 14 pacientes se realizaron cambios en los componentes plásticos de los ataches. El seguimiento clínico medio fue de 82,5 meses (60-102 meses).

Conclusiones. Los resultados del presente estudio indican que el tratamiento con implantes dentales en pacientes diabéticos es una terapéutica exitosa sin diferencias entre los pacientes diabéticos y no diabéticos.

PALABRAS CLAVE: Implantes dentales, pacientes geriátricos, diabetes mellitus, sobredentaduras, oseointegración, implantología oral.

\section{ABSTRACT}

Introduction. Implant dentistry can to constitute a therapeutic modality in the prosthodontic treatment of medically compromised patients. This study reports the evaluation of treatment with implants in patients with diabetes compared with patients without diabetes.

Methods. 48 edentulous patients (24 diabetic patients and 24 non-diabetic patients) were treated with 96 Galimplant $®$ sand-blasted and acid-etched surface implants for prosthodontic rehabilitation with mandibular overdentures. Two implants were inserted in each patient. Implants were loaded after a healing free-loading

* $\quad$ Profesor Asistente Honorario de Odontología Integrada de Adultos y de Gerodontología. Profesor del Máster de Implantología Oral. Facultad de Odontología. Universidad de Sevilla.

* * Profesor Asociado de Odontología Integrada de Adultos y Gerodontología. Profesor del Máster de Implantología Oral. Facultad de Odontología. Universidad de Sevilla

*** Profesor Titular de Odontología Integrada de Adultos y Gerodontología. Director del Máster de Implantología Oral. Facultad de Odontología. Universidad de Sevilla. 
period between 6 weeks with locator attaches. Clinical findings (implant and prosthodontics) were followed during at 7 years.

Results. Clinical results indicate a survival rate of implants of $95.8 \%$ in both groups. Four implant was lost during the follow-up period (two implants in each group). Media marginal bone loss was $0.7 \mathrm{~mm}$ in both groups. $100 \%$ of patients were treated with overdentures retained with 2 implants with locator attaches. Changes in plastic components of attaches were reported in 14 patients. The media follow-up was of 82.5 months (60-102 months).

Conclusions. Clinical results of this study indicate that treatment with dental implants in diabetic patients is a successful implant treatment without differences with nondiabetic patients.

KEY WORDS: Dental implants, geriatric patients, diabetes mellitus, overdentures, osseointegration, implant dentistry.

Fecha de recepción: 16 de marzo de 2020

Fecha de aceptación: 25 de marzo de 2020

JL Rondon Romero, I Ortiz Garcia, A Jimenez Guerra, N Matos Garrido, A España Lopez, L Monsalve Guil, E Velasco Ortega. El tratamiento con implantes en pacientes con diabetes. Un estudio comparativo a 7 años. 2020; 36, (2): 81-88.

\section{INTRODUCCIÓN}

El envejecimiento de la población ha provocado un aumento de la diabetes en los adultos mayores. La diabetes es una enfermedad crónica progresiva que cursa con hiperglucemia y un pobre control de la glucemia, cuya prevalencia se incrementa con la edad. La diabetes en los pacientes mayores cursa con mayor morbilidad que en los adultos más jóvenes con nefropatías, disfunción cognitiva, problemas cardiacos, deterioro visual por retinopatía, y otras complicaciones (fracturas, depresión, amputaciones de miembros, etc ${ }^{1-2}$. Además, las tasas de mortalidad de los pacientes con una diabetes de corta duración es significativamente mayor en los grupos de edad avanzada ${ }^{3}$. El tratamiento integral de la diabetes en los mayores comprende cambios en el estilo de vida (ej. ejercicio, control de la obesidad) y la prescripción de fármacos como la metformina y la insulina. La metformina sigue siendo el fármaco de primera elección por su eficacia, seguridad, tolerancia, vía de administración y coste económico ${ }^{3-4}$.

La diabetes está relacionada con diversas enfermedades dentales como la caries, la gingivitis y la periodontitis. La caries dental es más común en los pacientes diabéticos como consecuencia de cambios en la dieta y una mayor disminución del flujo salival, además de una deficiente higiene oral. Los pacientes con diabetes mal controlada padecen más frecuentemente una periodontitis avanzada que se incrementa con el consumo de tabaco ${ }^{5}$. Así mismo, la diabetes favorece la colonización por cándidas en la mucosa oral, sobre todo en pacientes mayores polimedicados, portadores de prótesis completa y xerostomía ${ }^{6}$.

La diabetes ha sido considerada una contraindicación relativa para el tratamiento con implantes dentales. La supervivencia a largo plazo de los implantes depende de la oseointegración y del mantenimiento de los tejidos periimplantarios. En este sentido, como la diabetes se ha relacionado con una cicatrización tisular retrasada, una enfermedad microvascular y una respuesta peor a la infección, se ha indicado como un factor de riesgo para el tratamiento con implantes ${ }^{7-8}$. Sin embargo, la evidencia científica reciente indica que las tasas de éxito de los implantes en pacientes diabéticos bien controlados pueden ser similares a los pacientes no diabéticos ${ }^{9-10}$. El control de la glucemia constituye la primera consideración importante en la planificación del tratamiento con implantes en los pacientes con diabetes, ya que existe una relación directa entre el nivel de glucosa en sangre y el desarrollo de complicaciones macro y microvasculares. Además, la hiperglucemia tisular afecta adversamente al sistema inmune alterando la respuesta a la cicatrización y como consecuencia a la oseointegración ${ }^{7,11-13}$. De hecho, en los pacientes diabéticos mal controlados con tasas 
elevadas de glucemia y de hemoglobina A1c, las complicaciones infecciosas como la periimplantitis son más frecuentes y las tasas de fracasos de los implantes son más elevadas ${ }^{11-13}$.

El objetivo del presente estudio era la valoración clínica del tratamiento con sobredentaduras mandibulares en pacientes edéntulos totales con diabetes comparada con pacientes edéntulos totales no diabéticos.

\section{PACIENTES Y METODOS.}

El presente estudio ha sido realizado por profesores de las unidades docentes de Odontología Integrada de Adultos y de Gerodontología y del Máster de Implantología Oral de la Facultad de Odontología de la Universidad de Sevilla.

Todos los pacientes seleccionados eran adultos mayores de ambos sexos y edéntulos totales. Previamente a la realización del estudio, se distribuyeron los pacientes en dos grupos, diabéticos y no diabéticos. Todos los pacientes seleccionados en el estudio fueron informados del protocolo del tratamiento con implantes, de los aspectos quirúrgicos y prostodóncicos, así como de la posible existencia de complicaciones y pérdida de implantes. Los pacientes autorizaron el tratamiento implantológico mediante un consentimiento informado. Antes del tratamiento, todos los pacientes fueron evaluados radiológicamente, con una ortopantomografía, y en los casos de reabsorción mandibular extrema con una tomografía computarizada de haz cónico.

CIRUGIA. Una hora antes de la cirugía, los pacientes comenzaron un régimen antibiótico preventivo (amoxicilina + clavulánico) durante una semana. Todos los pacientes recibieron anestesia local. Un total de 2 implantes Galimplant (B) (Galimplant, Sarria, España) de conexión externa y con superficie arenada y grabada fueron insertados en cada paciente entre los agujeros mentonianos y todos fueron estables después de la inserción. La preparación del lecho y la inserción de los implantes se realizó según el protocolo estandarizado a una velocidad constante de 800 r.p.m (Figuras 1 y 2). A los pacientes se les recomendó el enjuague diario con clorhexidina durante los primeros 30 días.

PROSTODONCIA. Se realizó la carga funcional precoz de los implantes a las 6 semanas de su

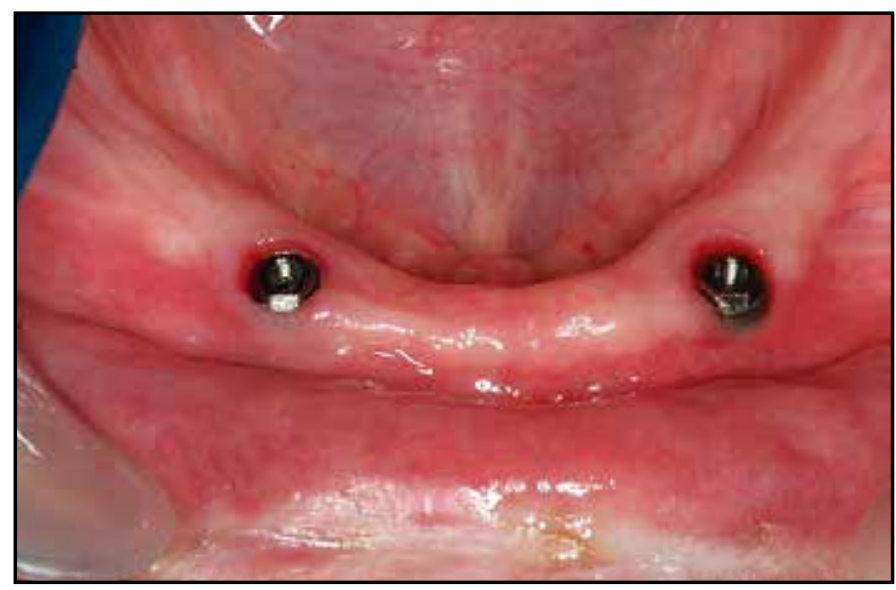

Figura 1. Aspecto clínico de los implantes insertados en un paciente diabético.

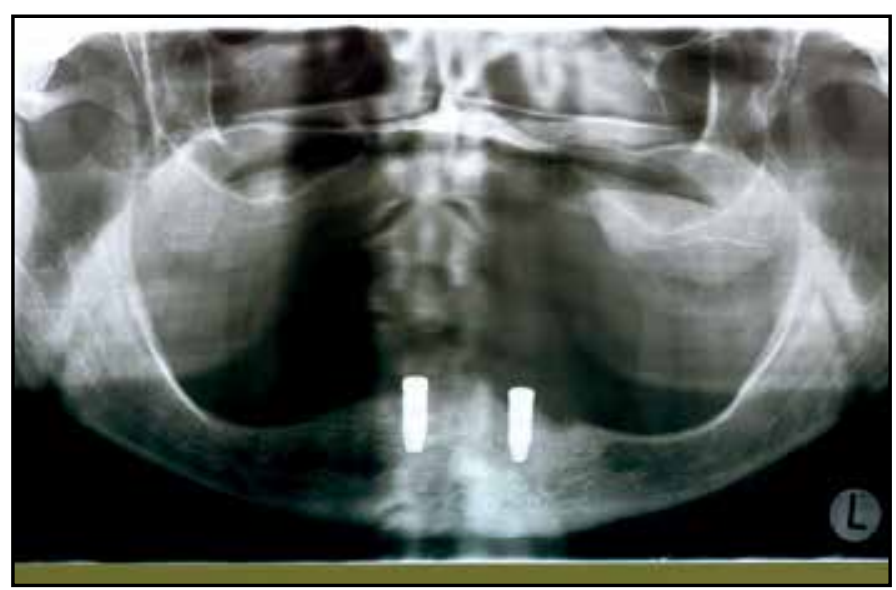

Figura 2. Imagen radiológica de los implantes insertados.

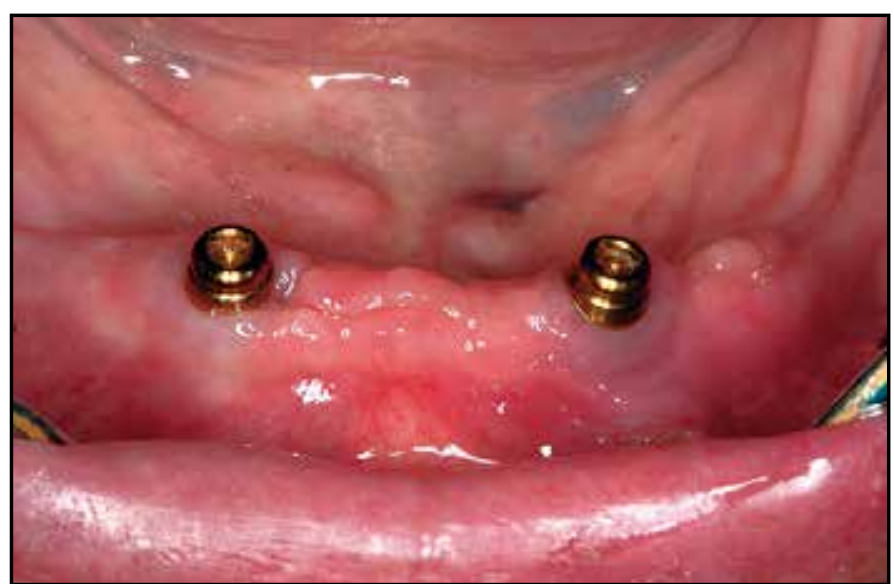

Figura 3. Colocación de los ataches sobre los implantes a las 6 semanas.

inserción (Figura 3). Los pacientes edéntulos totales no usaron sus prótesis completas durante 2 semanas después de la inserción de los implantes. A las 6 semanas, después de la inserción de los implantes, se realizó la carga funcional de los implantes mediante la colocación de una sobre- 
dentadura con ataches de fricción Overdent $®$ (Galimplant, Sarria, España).

SEGUIMIENTO CLINICO. El tiempo transcurrido de seguimiento clínico desde la carga funcional de todos los implantes fué al menos de 60 meses. Se realizaron revisiones clínicas de los pacientes, valorando el estado de los implantes y de las sobredentaduras. Se evaluó la pérdida de hueso periimplantario con radiografías periapicales.

ANALISIS ESTADISTICO. Se ha realizado una estadística descriptiva de los hallazgos clínicos del estudio, con referencia a las variables demográficas de los pacientes, los implantes insertados, las sobredentaduras realizadas, así como el éxito y las complicaciones del tratamiento. Todas las variables anteriores fueron analizadas estadísticamente. Las variables cualitativas fueron analizadas según el test de la chi-cuadrado, mientras que las variables cuantitativas fueron analizadas según el test de la varianza.

\section{RESULTADOS}

PACIENTES. 48 pacientes edéntulos totales portadores de una prótesis completa (26 hombres y 22 mujeres) participaron en el estudio (Tabla 1).

Diabéticos. 24 pacientes eran diabéticos tipo II, 14 hombres y 10 mujeres. La edad media de los pacientes con diabetes fué de 67,5 años (rango: $60-82$ años). 10 pacientes eran fumadores (41,7\%). 10 pacientes $(41,7 \%)$ presentaban otras patologías sistémicas (ej. hipertensión, insuficiencia cardiaca)(Tabla 1).

No diabéticos. 24 pacientes no eran diabéticos, de los que 12 eran hombres y 12 eran mujeres. La edad media de los pacientes con diabetes fué de 69,3 años (rango: $60-80$ años). 12 pacientes eran fumadores $(50 \%)$. 20 pacientes $(83,3 \%)$ presentaban otras patologías sistémicas (ej. hipertensión, insuficiencia cardiaca)(Tabla 1).

No hubo diferencias significativas entre los dos grupos de pacientes diabéticos y no diabéticos en relación a la edad, sexo, consumo de tabaco y otros antecedentes médicos(test de la chi-cuadrado; $\mathrm{p}=$ 0,$67108 ; \mathrm{p}=0,68187 ; \mathrm{p}=0,68839$ 。 $\mathrm{p}=0,31731$ ).

IMPLANTES. Se insertaron un total de 96 implan-

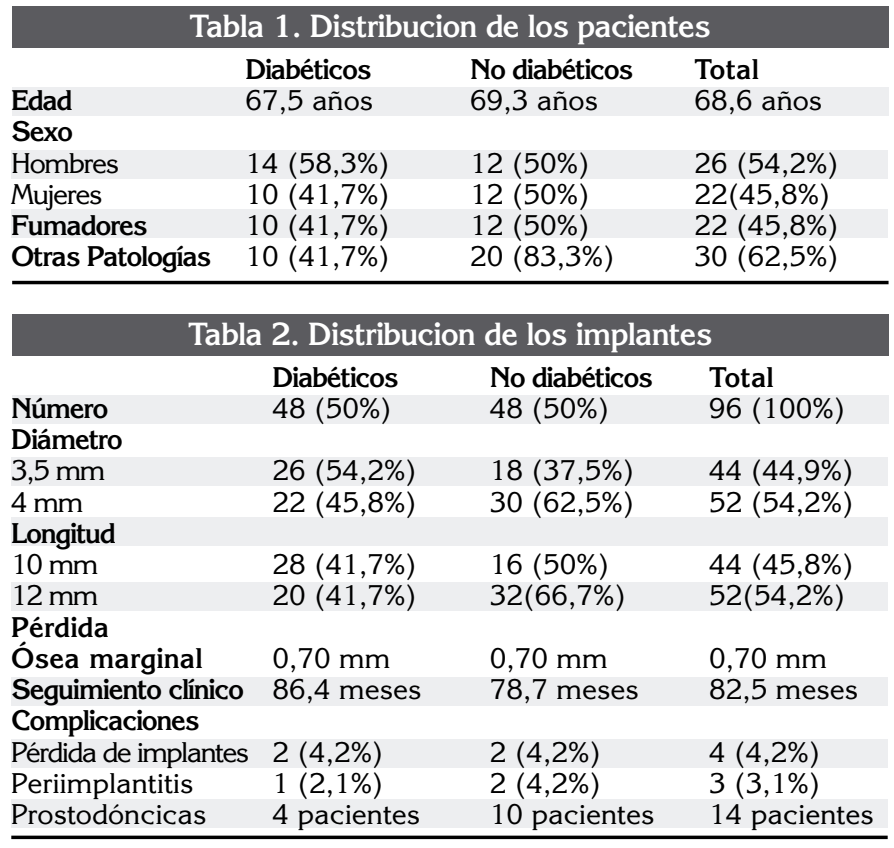

tes (48 implantes en los diabéticos y 48 implantes en los pacientes no diabéticos) en la mandíbula edéntula, de los correspondientes 48 pacientes (Tabla 2). El 100\% de los implantes fueron insertados de forma diferida, tras un periodo mínimo de 6 meses después de la última extracción dental.

Diabéticos. De los 48 implantes insertados en los pacientes, 26 implantes $(54,2 \%$ ) eran de $3,5 \mathrm{~mm}$ de diámetro y 22 implantes $(45,8 \%)$ eran de $4 \mathrm{~mm}$ de diámetro; mientras que 28 implantes $(58,3 \%)$ eran de $10 \mathrm{~mm}$ de longitud y 20 implantes $(41,7 \%)$ eran de $12 \mathrm{~mm}$ de longitud. La pérdida ósea marginal fué de 0,70 mm (rango: 0,3-1,4 mm)(Tabla 2)

No diabéticos. De los 48 implantes insertados en los pacientes, 18 implantes $(37,5 \%)$ eran de 3,5 $\mathrm{mm}$ de diámetro y 30 implantes (62,5\%) eran de $4 \mathrm{~mm}$ de diámetro; mientras que 16 implantes $(33,3 \%)$ eran de $10 \mathrm{~mm}$ de longitud y 32 implantes $(66,7 \%)$ eran de $12 \mathrm{~mm}$ de longitud. La pérdida ósea marginal fué de 0,70 mm (rango: 0,4-1,4 $\mathrm{mm}$ )(Tabla 2).

No hubo diferencias significativas entre los dos grupos de pacientes diabéticos y no diabéticos en relación al diámetro y longitud de los implantes (test de la chi-cuadrado; $\mathrm{p}=0,40637 ; \mathrm{p}=$ 0,21653 ). No hubo diferencias significativas entre los dos grupos de pacientes diabéticos y no diabéticos en relación a la pérdida ósea marginal media (test de varianza; ANOVA $p=0,8460$ ). No 
hubo diferencias significativas entre el consumo de tabaco y la pérdida ósea marginal media (test de varianza; ANOVA $p=0,3247$ ).

PROSTODONCIA. En todos los pacientes se realizó la carga funcional precoz de los implantes mediante una sobredentadura con 2 retenedores de fricción. En este sentido, se realizaron un total de 48 sobredentaduras implantorretenidas. El seguimiento clínico medio de los pacientes estudiados ha sido de 82,5 meses (rango: 60 meses- 102 meses).

En los pacientes diabéticos el seguimiento clínico medio fue de 86,4 meses (rango: 73-104 meses). En los pacientes no diabéticos el seguimiento clínico medio fue de 78,7 meses (rango: 60-104 meses). No hubo diferencias significativas entre los dos grupos de pacientes diabéticos y no diabéticos en relación al número de meses de seguimiento clínico (test de la chi-cuadrado; $\mathrm{p}=0,08300$ ).

COMPLICACIONES. Se perdieron 4 implantes $(4,2 \%)$ en 4 pacientes $(8,4 \%)$, que después de 60 días se sustituyeron por otros 4 implantes. Hubo 3 casos de periimplantitis $(3,1 \%)$ en 3 pacientes (6,2\%). En 14 pacientes (29,2\%) hubo complicaciones prostodóncicas relacionadas con los ataches que fueron sustituidos (Tabla 3 ).

Diabéticos. Se perdieron 2 implantes (2,1\%) en 2 pacientes $(4,2 \%)$. Los dos pacientes eran fumadores. En 14 pacientes hubo complicaciones prostodóncicas relacionadas con los ataches que fueron sustituidos. Hubo un caso de periimplantitis $(2,1 \%)$ en un paciente (2,1\%). En 4 pacientes $(16,7 \%)$ hubo complicaciones prostodóncicas relacionadas con los ataches, que fueron sustituidos.

No diabéticos. Se perdieron 2 implantes (2,1\%) en 2 pacientes $(8,3 \%)$. Los dos pacientes eran fumadores. Hubo 2 casos de periimplantitis $(4,2 \%)$ en 2 pacientes (8,3\%). En 10 pacientes $(41,7 \%)$ hubo complicaciones prostodóncicas relacionadas con los ataches, que fueron sustituidos.

No hubo diferencias significativas entre los dos grupos de pacientes diabéticos y no diabéticos en relación a la pérdida de implantes, periimplantitis y complicaciones prostodóncicas (test de la chi-cuadrado; $p=1,0000 ; p=0,13159 ; p=$ 0,31731). La relación entre pérdida de implantes y consumo de tabaco no fué significativa (test de la chi-cuadrado; $\mathrm{p}=0,12485)$.

\section{DISCUSIÓN}

La rehabilitación con sobredentaduras con implantes en adultos mayores representa una opción de tratamiento muy favorable para conseguir una grado aceptable de función oral y de estética que favorezca una mejora en la calidad de vida de los pacientes geriátricos. Las sobredentaduras mandibulares han demostrado su eficacia clínica mediante la colocación $\mathrm{de}^{2-4}$ implantes en el sector anterior interforaminal con diversos protocolos de carga convencional, precoz e inmediata $^{14}$.

La terapéutica con implantes dentales en los pacientes mayores requiere una valoración de sus antecedentes médicos que puedan jugar un papel importante en el tratamiento. Aunque la edad avanzada no constituye un criterio de inclusión o exclusión del protocolo con implantes dentales, es importante determinar el grado de salud general que puede contraindicar relativa o absolutamente el tratamiento con implantes, cuando existe un compromiso médico grave o incapacitante. Los pacientes geriátricos con un estado físico o psíquico deteriorado no son buenos candidatos para un tratamiento con implantes dentales ${ }^{15-16}$.

En el presente estudio, el $41,7 \%$ de los pacientes diabéticos y el $83,3 \%$ de los pacientes sin diabetes presentaban otras patologías sistémicas (ej. hipertensión, insuficiencia cardiaca), lo que confirma la importancia de la valoración sistémica y farmacológica de los pacientes que van a ser tratados con implantes. En este sentido, los hallazgos clínicos del estudio demuestran que los antecedentes médicos no influyeron en el tratamiento con implantes clínicos cuando estas enfermedades están bien controladas por el médico general o el geriatra ${ }^{5,11}$.

El hábito de fumar constituye un factor de riesgo importante en el éxito de los implantes. El tabaco se ha relacionado con una mayor incidencia de fracasos de implantes y problemas periimplantarios ${ }^{17}$. En el presente estudio, el $41,7 \%$ de los pacientes diabéticos y el $50 \%$ de los pacientes sin diabetes eran fumadores. El $100 \%$ de los implantes perdidos se observaron en pacientes diabéticos y no diabéticos fumadores. Además, los pacientes fumadores presentaron una mayor pérdida de hueso marginal que los pacientes no fumadores $(0,76 \mathrm{~mm}$ vs $0,68 \mathrm{~mm})$. En este sentido, algunos estudios realizados en pacientes tratados con sobredentaduras han confirmado que el tabaco incrementó la perdida ósea margi- 
nal y la incidencia de periimplantitis en los implantes insertados en los pacientes fumadores, en comparación con los implantes de los no fumadores ${ }^{18-19}$.

Los resultados globales del presente estudio demuestran que mediante un protocolo clínico integral que englobe los diversos procedimientos quirúrgicos y prostodóncicos, se puede conseguir y mantener una oseointegración favorable de los implantes a largo plazo tanto en los pacientes diabéticos como en los no diabéticos. En este sentido, el tratamiento de los pacientes geriátricos edéntulos. con implantes mediante sobredentaduras mandibulares del presente estudio, constituye una técnica predecible obteniendo una supervivencia de los implantes del $95,8 \%$ en ambos grupos con y sin diabetes, durante un periodo de 7 años.

La revisión de la literatura de estudios con implantes realizados en pacientes diabéticos, fundamentalmente en adultos con diabetes tipo II, muestran unas tasas de supervivencias similares a los implantes insertados en pacientes sanos, entre el 86$100 \%$ durante el primer año después de la cirugía, aunque parece haber un incremento de los fracasos de los implantes conforme aumenta el periodo de seguimiento clínico $7,9,11,20-21$. Un estudio prospectivo a corto plazo con 50 implantes en 35 pacientes con diabetes demuestra una tasa de supervivencia del $100 \%$, lo que indica que cuando se realiza un control metabólico y bioquímico adecuado de la glucemia y de la $\mathrm{HbA} 1 \mathrm{c}$ en los pacientes diabéticos disminuyen las complicaciones inmediatas y no se afecta el éxito del tratamiento ${ }^{22}$. Resultados similares son mostrados en un estudio reciente comparativo entre 15 pacientes diabéticos tipo 2 y 14 sujetos sanos donde las tasas de supervivencia y éxito de los implantes fueron similares en ambos grupos despues de 6 meses de la carga funcional con las correspondientes restauraciones ${ }^{23}$.

A más largo plazo, las expectativas de supervivencia de los implantes pueden ser menores. En este sentido, un estudio retrospectivo a 6,5 años de seguimiento con 215 implantes colocados en 40 pacientes diabéticos presenta una pérdida del $14,4 \%$ de los implantes, la mayoría durante el primer año después de la carga funcional, lo que demuestra una tasa de supervivencia del 85,6\% 24. Un estudio retrospectivo valora a 169 pacientes diabéticos tipo 2 moderadamente controlados obtenidos de una revisión de 3256 historias clínicas con 1.112 implantes colocados durante un periodo medio de seguimien- to de 8,7 años. La tasa de supervivencia fué del $94 \%$ ya que 67 implantes fracasaron. No hubo diferencias con los pacientes no diabéticos ${ }^{25}$.

La diabetes mellitus puede influir en el tratamiento con implantes durante la fase quirúrgica con la inserción de los implantes y también en la realización de la segunda cirugía en la técnica sumergida con la colocación de los pilares de cicatrización. El implantólogo debe enfocar previamente el tratamiento con un control de la glucemia y de la HbA1c en contacto con su médico especialista, y debe prescribir un régimen antibiótico previo a la cirugía y aplicación de un gel o colutorio de clorhexidina $a^{5,20}$.

Posteriormente en la fase de mantenimiento, la diabetes mellitus puede influir negativamente en la respuesta fisiológica (ej. pérdida ósea marginal) o patológica (mucositis/periimplantitis) de los implantes dentales ${ }^{10,12,26-27}$. En el presente estudio la pérdida ósea marginal $(0,7 \mathrm{~mm})$ fué similar en ambos grupos de pacientes diabéticos y no diabéticos se observó una baja incidencia de periimplantitis $(3,1 \%)$. La revisión de la literatura sugiere que los pacientes con diabetes tipo 2 tratados con implantes tienen mayor tendencia a desarrollar pérdida de hueso marginal y periimplantitis ${ }^{12,27}$. En este sentido, la intensidad de los cambios periimplantarios puede estar relacionada con el control de la glucemia y la cuantificación de la $\mathrm{HbA} 1 \mathrm{c}$, aunque existe suficiente evidencia de que los pacientes con diabetes tipo 2 que acuden a sus revisiones periódicas tanto médicas como implantológicas ofrecen una respuesta tisular periimplantaria muy favorable ${ }^{23,28}$. Un estudio longitudinal compara los aspectos clínicos periimplantarios (ej. sondaje, pérdida ósea marginal, sangrado), biomarcadores (ej. interleukinas, osteoprotegerina) y microbiológicos (ej. Prevotella intermedia, Porphyromonas gingivalis) de pacientes diabéticos adultos tratados con implantes comparados con pacientes sanos durante 1 año de seguimiento ${ }^{10}$. Las conclusiones del estudio indican que cuando los pacientes diabéticos mantienen un control metabólico adecuado y un programa de revisiones periódicas, no existen diferencias con los pacientes sanos ${ }^{10}$.

Los resultados del presente estudio demuestran que el tratamiento de los pacientes diabéticos con sobredentaduras constituye un alternativa implantológica con éxito en la rehabilitación de los pacientes mayores edéntulos totales. En este sentido, en el presente estudio ninguna prótesis ha tenido que ser sustituida y solamente se han cambiado los retenedores plás- 
ticos en el $30 \%$ de los pacientes. Estos hallazgos clínicos se corresponden con los obtenidos en algunos estudios realizados sobre el tratamiento con sobredentaduras en pacientes diabéticos 29-30. Un estudio prospectivo a 1 año, analizó la influencia del control de la enfermedad (niveles de HbA1c) en el tratamiento con implantes en pacientes diabéticos tipo 2 29. Se trataron 110 pacientes edéntulos con sobredentaduras mandibulares con dos implantes. Las tasas de supervivencia fueron del 93\%, 92,6\% y del 95\% para los implantes colocados en pacientes sin diabetes, pacientes con un buen control y pacientes con un control pobre de la diabetes 29. Un estudio prospectivo a 2 años valora los resultados clínicos de sobredentaduras mandibulares con dos implantes retenidas con ataches magnéticos en pacientes diabéticos tipo 2 29. En 28 pacientes se insertaron 56 implantes. Los implantes fueron examinados a los 6,12 y 24 meses. Los índices de placa, gingival, la profundidad de sondaje y la pérdida de hueso marginal se incrementaron durante el periodo de seguimiento. La estabilidad de los implantes (ISQ) descendió después de los 6 meses. La tasa de supervivencia fué del $91 \% 30$.

\section{CONCLUSIONES}

El tratamiento con implantes en pacientes con diabetes controlada puede realizarse con un éxito elevado similar a los pacientes sin diabetes. En este sentido, las sobredentaduras con implantes representan una opción favorable en los pacientes edéntulos geriátricos mandibulares diabéticos cuando se realiza un protocolo quirúrgico y prostodóncico adecuado y se mantiene a los pacientes en un programa de revisiones periódicas.

\section{BIBLIOGRAFIA}

1. Valencia WM, Botros D, Vera-Nunez $M$, Dang $S$. Diabetes treatment in the elderly: incorporating geriatrics, technology, and functional medicine. Curr Diab Rep 2018; 18: 95.

2. Kirkman MS, Briscoe VJ, Clark N, Florez H, Haas LB, Halter JB, et al. Diabetes in older adults. Diabetes Care 2012; 35:2650-64.

3. Chou MY, Huang ST, Liang CK, Hsiao FY, Chen LK. Clinical characteristics and 10-year outcomes of diabetes in adults with advancing age at onset: A population cohort. Arch Gerontol Geriat 2020; 88: 104039.

4. Thrasher J. Pharmacologic management of type 2 diabetes mellitus: available therapies. Am J Med 2017; 130: 4-17.

5. Tavares M, Lindefjeld Calabi KA, San Martin L. Systemic diseases and oral health. Dent Clin N Am 2014; 58: 797-814.

6. Pedersen AML, Nauntofte B, Smidt D, Torpet LA. Oral mucosal lesions in older people: relation to salivary secretion, systemic diseases and medications. Oral Dis 2015; 21:721-729.

7. Chrcanovic BR, Albrektsson T, Wennerberg A. Diabetes and oral implant failure: a systematic review. J Dent Res 2014; 93: 859-867.

8. Almehmadi AH. Awareness of population regarding the effects of diabetes on dental implant treatment in Jeddah, Saudi Arabia. Heliyon 2019; 5:02407.

9. Arbildo H, Lamas C, Camara D, Vásquez $\mathrm{H}$. Dental implant survival rate in well-controlled diabetic patients. A systematic review. J Oral Res 2015; 4: 404-410.

10. Tatarakis N, Kinney JS, Inglehart M, Braun TM, Shelburne C, Lang NP, Giannobile WV, Oh T-J. Clinical, microbiological, and salivary biomarker profiles of dental implant patients with type 2 diabetes. Clin Oral Impl Res 2014; 25: 803-812.

11. Schimmel M, Srinivasan M, McKenna G, Müller $F$. Effect of advanced age and/or systemic medical conditions on dental implant survival: A systematic review and meta-analysis. Clin Oral Impl Res 2018; 29(Suppl. 16):311-330.

12. Chambrone L, Palma LF. Current status of dental implants survival and peri-implant bone loss in patients with uncontrolled type-2 diabetes mellitus. Curr Opin Endocrinol Diabetes Obes 2019; 26:219-222.

13. Naujokat H, Kunzendorf B, Wiltfang J. Dental implants and diabetes mellitus. A systematic review. Int J Impl Dent 2016; 2:5.

14. Velasco E, Medel R, García A, Ortiz I, España 
A, Nuñez E. Sobredentaduras con implantes en pacientes geriátricos edéntulos totales. Av Odontoestomatol 2015; 31: 161-172.

15. Schimmel M, Müller F, Suter V, Buser D. Implants for elderly patients. Periodontology 2000 2017; 73: 228-240.

16. Velasco E, Monsalve L, Jiménez A, Segura JJ, Matos N, Moreno J. El tratamiento con implantes en los pacientes adultos mayores. Av Odontoestomatol2015; 31: 219-227.

17. Cavalcanti R, Oreglia F, Manfredonia MF, Gianserra $R$, Esposito $M$. The influence of smoking on the survival of dental implants: a 5-year pragmatic multicentre retrospective cohort study of 1727 patients. Eur J Oral Implantol 2011; 4: 3945.

18. Stoker G, van Waas R, Wismeijer D. Long-term outcomes of three types of implant-supported mandibular overdentures in smokers. Clin Oral Implants Res 2012; 23: 925-929.

19. Offord D, Mathieson G, Kingsford N, Matthys C, Glibert M, De Bruyn H. Peri-implant health, clinical outcome and patient-centred outcomes of implant-supported overdentures in the mandible and the maxilla. BDJ Open 2017; 3: 17017.

20. Katyayan PA, Katyayan M, Shah RJ. Rehabilitative considerations for dental implants in the diabetic patient. J Indian Prosthodont Soc 2013; 13:175-183.

21. Oates TW, Huynh-Ba G, Vargas A, Alexander P, Feine J. A critical review of diabetes, glycemic control, and dental implant therapy. Clin Oral Implants Res 2013; 24:117-127.

22. Dowell S, Oates TW, Robinson M. Implant success in peoplewith type $2 \mathrm{~d}$ iabetes mellitus with varying glycemic control: A pilot study. J Am Dent Assoc 2007; 138:355-361.

23. Cabrera-Domínguez J, Castellanos-Cosano L, Torres-Lagares D, Machuca- Portillo G. A prospective case-control clinical study of titaniumzirconium alloy implants with a hydrophilic surface in patients with type 2 diabetes mellitus. Int J Oral Maxillofac Implants 2017; 32:1135-1144.
24. Fiorellini JP, Chen PK, Nevins M, Nevins ML. A retrospective study of dental implants in diabetic patients. Int J Perio Rest Dent 2000; 20:366-373.

25. Ormianer Z, Block J, Matalon S, Kohen J. The effect of moderately controlled type 2 diabetes on dental implant survival and peri-implant bone loss: a longterm retrospective study. Int $\mathrm{J}$ Oral Maxillofac Implants 2018; 33:389-394.

26. Aguilar-Salvatierra A, Calvo-Guirado JL, Gonzalez-Jaranay M, Moreu G, Delgado-Ruiz RA, Gomez-Moreno G. Periimplant evaluation of immediately loaded implants placed in esthetic zone in patients with diabetes mellitus type 2: A two-year study. Clin Oral Impl Res 2016; 27: 156-161.

27. SoutoMaior JR, Pellizzer EP, Gomes JML, Lemos CAA, Santiago Jr JF Vasconcelos BCE, Moraes SLD. Influence of diabetes on the survival rate and marginal bone loss of dental implants: an overview of systematic reviews. J Oral Implantol 2019; 45:334-340.

28. Gómez-Moreno G, Aguilar-Salvatierra A, Rubio Roldán J, Guardia J, Gargallo J, Calvo-Guirado JL. Peri-implant evaluation in type 2 diabetes mellitus patients: a 3-year study. Clin Oral Impl Res 2015; 26: 1031-1035.

29. Oates Jr TW, Galloway P, Alexander P, Vargas Green A, Huynh-Ba G, Feine J, et al. The effects of elevated hemoglobin $A(1 c)$ in patients with type 2 diabetes mellitus on dental implants: survival and stability at one year. J Am Dent Assoc 2014; 145:1218-1226.

30. Shawky AF, Ashour EM. Unsplinted implants retaining a mandibular overdenture with magnetic attachments: a short-term clinical study in controlled type 2 diabetic patients. Quintessence Int 2014; 45:299-305.

\section{AUTOR DE CORRESPONDENCIA:}

Prof. Eugenio Velasco Ortega

Facultad de Odontología

$\mathrm{C} /$ Avicena $\mathrm{s} / \mathrm{n}$

Tfno: 954481132 e-mail: evelasco@us.es

41009 Sevilla 